\section{Psoriasis: the plot}

\section{thickens... .}

\section{Michael P. SChÖn ${ }^{1}$ And Thomas RuZICKA ${ }^{2}$}

${ }^{1}$ Department of Dermatology, Otto von Guericke University, Leipzigerstrasse 44,39120 M agdeburg, Germany. (M ichael.Schoen@ M edizin.Uni-

E M agdeburg.DE) ${ }^{2}$ Department of Dermatology, $\mathrm{H}$ einrich-H eine-University,

Oั M oorenstrasse 5,40225 Düsseldorf, Germany

Few diseases illustrate the central role of

chemokines in tissue-specific leukocyte recruit-

ment as vividly as psoriasis, one of the most

ह common chronic inflammatory skin disorders.

Esoriasis is primarily a T cell-mediated

autoimmune disease ${ }^{1}$. In addition, there is a

prominent infiltration of neutrophils within the

dermis that is focused within epidermal

윽 microabscesses, mast cells within the dermis

은 and diffusely distributed macrophages. Because

its complex tissue alterations require careful

orchestration of sequential and highly specific

@ leukocyte functions, psoriasis may serve as a

흑 model disease for studying the intertwined

$\mathbf{Q}$ interactions of immigrating immune cells with

을 resident epithelial and mesenchymal cells. The

疍 microenvironmental regulation of these patho-

$\mathbf{z}$ genic events, however, remains unknown. An

$\overline{-}$ exciting new concept is now emerging that

N highlights a pivotal role of chemokines in traf-

(2) ficking, adhesion, subtype-specific influx and

compartmentalization of leukocytes in the pso-

- riatic disease process ${ }^{2}$ (Fig. 1).

As the chemokines TARC and MDC are expressed by the cutaneous vasculature and their receptor, CCR4, is expressed by some circulating $\mathrm{CLA}^{+}$memory $\mathrm{T}$ cells, these chemokines presumably contribute to the preferential recruitment of skin-homing memory T cells via stimulation of integrin-ICAM-1 adhesive interactions ${ }^{3}$. CTACK (CCL27), a ligand for the orphan chemokine receptor GPR-2 (CCR10), is constitutively expressed by epidermal keratinocytes but can be increased by TNF- $\alpha$ and IL- $1 \beta^{4}$. Because CCR10 is expressed by T cells and skinderived Langerhans cells, CTACK appears to contribute to epidermal localization of these cells ${ }^{4}$. In addition, MIP-3 $\alpha$ (CCL20) colocalizes in psoriatic epidermis with epidermal $\mathrm{T}$ cells and skin-homing CLA $^{+} \mathrm{T}$ lymphocytes express high amounts of the MIP-3 $\alpha$ receptor, $\mathrm{CCR}^{5}$. Psoriatic $\mathrm{CLA}^{+} \mathrm{T}$ cells respond to lower concentrations of MIP- $3 \alpha$, as compared to $\mathrm{T}$ cells from normal donors. In addition, MIP-3 $\alpha$ can be induced on keratinocytes by proinflammatory cytokines involved in the pathogenesis of psoriasis ${ }^{5}$.

The CXC-chemokine, MIG, is expressed exclusively on a spatially restricted subpopulation of endothelial cells and macrophages within the papillary dermis directly underneath the hyperplastic psoriatic epidermis ${ }^{6}$. As MIG is a T cell-attracting chemokine, it may contribute to epidermal $\mathrm{T}$ cell localization, possibly via TGF- $\beta_{1}$-induced expression of the $\alpha_{\mathrm{E}}(\mathrm{CD} 103) \beta_{7}$ integrin. Given that MIG can be induced in macrophages and dermal microvascular endothelial cells by $\mathrm{T}$ cell-derived IFN- $\gamma$, a microenvironmental T cell-associated inflammation-boosting loop may contribute to the histopathological changes of psoriatic skin. This process may be augmented by the CC lymphocyte chemoattractant RANTES, which is up-regulated within psoriatic epidermis but not other inflammatory skin disorders ${ }^{7}$. Another loop exists with respect to the CC chemokine MCP-1, the expression of which is increased in psoriatic epidermis. MCP-1 induces its own production by monocytes (autocrine loop) and stimulates fibroblast expression of TGF- $\beta 1^{8}$, a cytokine thought to contribute to epidermal $\mathrm{T}$ cell localization via induction of the integrin $\alpha_{\mathrm{E}} \beta_{7}$ (K. Pauls and M. P. Schön, unpublished data). In addition, both MCP-1 and RANTES may attract mast cells to psoriatic skin ${ }^{7}$. Because the CXC chemokine, ITAC, can be induced by IFN- $\gamma$ on keratinocytes and endothelial cells ${ }^{9}$, it is conceivable that ITAC also contributes to cutaneous T cell local- ization in psoriasis.

The formation of epidermal neutrophil accumulations, called Munro's microabscesses, is a hallmark feature of psoriasis ${ }^{1}$. Interestingly, there is a high content of neutrophil-attracting chemokines including IL- 8 and GRO- $\alpha$ within psoriatic scales ${ }^{10}$, the product of the excessive keratinization within the psoriatic plaques. This results in a "vicious cycle" of neutrophil attraction and further tissue damage mediated by neutrophils themselves as well as other mechanisms. In addition, IL-8 down-regulates epidermal expression of the IL-10 receptor, which results in a loss of negative immunoregulatory stimuli in psoriatic lesions.

Keratinocyte hyperproliferation in psoriatic skin also appears to be induced, at least in part, by IL- 8 and GRO- $\alpha$ because their receptor, CXCR2, is overexpressed in psoriatic, but not normal, $\operatorname{skin}^{10}$.

Overall, recent advances in chemokine research have contributed tremendously to our understanding of the immunopathogenesis of psoriasis and, possibly, opened a new era of specific therapeutic interventions.

\footnotetext{
1. Christophers, E. Int.Arch.Allergy Immunol. 110, 199-206 (1996).

2. Zlotnik,A., Morales, J. \& Hedrick, J.A. Crit. Rev. Immunol. 19, 1-47

(1999).

3. Campbell, J. J. et al. Nature $\mathbf{4 0 0 , 7 7 6 - 7 8 0 ~ ( 1 9 9 9 ) . ~}$

4. Homey, B. et al. J. Immunol. 164, 3465-3470 (2000)

5. Homey, B. et al. J. Immunol. 164, 6621-6632 (2000)

6. Goebeler, M. et al. J. Pathol. 184, 89-95 (1998).

7. Raychaudhuri, S. P. et al. Acta Derm. Venereol. 79, 9-11 (1999).

8. Yamamoto,T., Eckes, B., Mauch, C., Hartmann, K. \& Krieg,T.

J. Immunol. 164,6174-6179 (2000).
160.,

9. Mazanet, M. M., N eote, K.\& Hughes, C. C.W. J. Immunol. 164 5383-5388 (2000)

10. Kulke, R. et al. J. Invest. Dermatol. 110, 90-94 (1998)
}

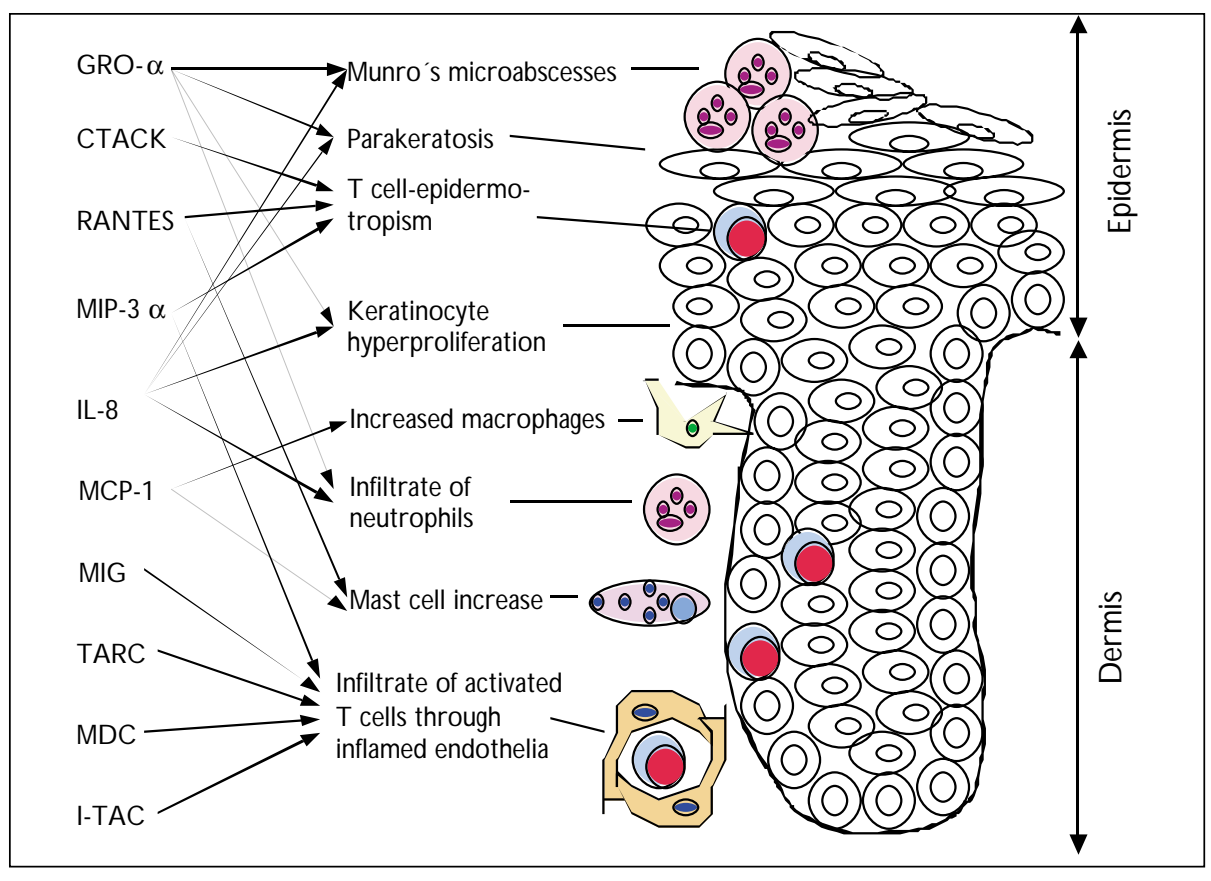

Figure 1. Synopsis of selected chemokine actions in psoriatic lesions. 